\title{
Variación morfológica de los peces Hemibrycon boquiae y Hemibrycon rafaelense (Characiformes: Characidae) en el Río Cauca, Colombia
}

\author{
César Román-Valencia ${ }^{1}$, Dahiana K. Arcila-Mesa ${ }^{1} \&$ Hernando Hurtado T. $^{2}$ \\ 1. Universidad del Quindío, Laboratorio de Ictiología, A.A. 2639, Armenia, Colombia; ceroman@uniquindio.edu.co; \\ arciladk@gmail.com \\ 2. Universidad del Quindío, Grupo de Investigación y Asesoría en Estadística, Armenia, Colombia.
}

Recibido 20-XI-2008. Corregido 22-II-2009. Aceptado 25-III-2009.

\begin{abstract}
Morphological variation in populations of Hemibrycon boquiae and H. rafaelense from Rio Cauca, Colombia. We analyzed the variation in morphological and osteological characteristics of Hemibrycon boquiae and Hemibrycon rafaelense in the Cauca River. The multivariate analysis determined populations of H. boquiae in nine streams: Boquier, Honda, Las Aguilas, Doña Juana, Villa Paola, Portachuelo, The Snake, Aimes and Ramirez, and populations of H. rafaelense in four streams: Canceles, Clara, San Rafael and San Jose. There were morphometric differences among populations of $H$. boquiae $(\mathrm{n}=277)$ and $H$. rafaelense $(\mathrm{n}=$ 121). Nevertheless, there were significant meristic discrepancies among populations of both species: number of scales between lateral line and anal fin, and number of scales between lateral line and pelvic fin. The absence of bilateral symmetry was observed in the number of maxillary teeth in $H$. boquiae. The cluster analysis distance for osteological characters, including the number of predorsal scales, separated $H$. boquiae and $H$. rafaelense in two defined groups. Rev. Biol. Trop. 57 (3): 541-556. Epub 2009 September 30.
\end{abstract}

Key words: morphology, distribution, Hemibrycon, Andes, tropical fish.

La selección divergente guía las diferencias fenotípicas a través de la diferenciación genética o plasticidad fenotípica. Otras fuentes de divergencia pueden actuar en cambios microevolutivos y guiar a la especiación. Sin embargo, el cruce entre poblaciones (migraciones o flujo génico) pueden imponer una diversificación adaptativa entre ambientes alternativos (Langerhans et al. 2003). En este contexto, las especies y poblaciones de Hemibrycon presentan un interesante modelo de distribución alrededor de la cuenca del Amazonas (Géry 1962, Román-Valencia et al. 2006, 2007, Bertaco et al. 2007, Arcila-Mesa 2008), para Colombia se distribuyen en alopátria (Román-Valencia et al. 2006, 2007, Román-Valencia \& ArcilaMesa 2008, Arcila-Mesa 2008). Se infiere que la historia geológica del sistema del Magdalena ha desempeñado un papel fundamental en los eventos de cladogénesis de las especies de Hemibrycon debido al aislamiento de sus poblaciones.

Hemibrycon es un grupo monofilético (Arcila-Mesa 2008), y H. boquiae se identifica para la quebrada Boquia (Eigenmann 1927, Román-Valencia 2001, Lima et al. 2003, Ortega-Lara et al. 2006). Román-Valencia (2001) realiza la redescripción de $H$. boquiae con base en material de la localidad típica (= quebrada Boquia), posteriormente se determinan poblaciones de esta especie en diferentes drenajes del alto y medio Cauca (véase material examinado en este trabajo) y se evidencian caracteres que diferencian las poblaciones pero no suficientes para diagnosticar nuevas especies. Román-Valencia \& Arcila-Mesa (2008) 
describen a $H$. rafaelense con base en caracteres merísticos y osteológicos, y al igual que $H$. boquiae se caracteriza por presentar todas sus poblaciones en alopátria (Arcila-Mesa 2008).

El objetivo de ese trabajo consiste en caracterizar las poblaciones de $H$. boquiae y de H. rafaelense en los drenajes del Río Cauca.

\section{MATERIALES Y MÉTODOS}

Se analizaron datos de las poblaciones de $H$. boquiae y de $H$. rafaelense de su localidad típica, y de diversas localidades a lo largo del sistema del alto y medio Cauca. Se examinó material depositado en: Instituto de Investigaciones Biológicas "Alexander Von Humboldt", Villa de Leyva, Boyacá, Colombia (IAvH); Unidad de Ictiología del Instituto de Ciencias Naturales, Universidad Nacional de Colombia, Bogotá, Colombia (ICNMNH); Laboratorio de Ictiología, Universidad del Quindío, Armenia, Colombia (IUQ).

Variables: Las medidas morfométricas se tomaron con calibrador digital, hasta centésimas de milímetro; todas las medidas fueron obtenidas punto a punto; los recuentos de escamas y radios se realizaron con estereoscopio; las medidas y recuentos se realizaron sobre el lado izquierdo de los ejemplares, excepto cuando éstos estaban deteriorados en tal lado. Para $H$. boquiae se realizaron conteos del número de dientes del maxilar en lado derecho e izquierdo. Las medidas y conteos siguen a Vari y Siebert (1990).

Las observaciones osteológicas se realizaron sobre ejemplares clareados y teñidos de acuerdo a las modificaciones del método descrito por Taylor \& Van Dyke (1985), Song \& Parenti (1995). Cuando fue posible se examinaron dos o seis ejemplares clareados y teñidos de cada población con el objetivo de visualizar caracteres asociados a variaciones anatómicas y dimorfismo sexual. La nomenclatura de huesos se basó en Weitzman (1962) y Vari (1995). Se incluyeron las cuatro primeras vértebras del aparato de Weber y el centrum terminal se contó como una vértebra. En total se examinaron ocho poblaciones de $H$. boquiae y cuatro de $H$. rafaelense provenientes de diferentes localidades del río Cauca.

Análisis estadístico: Se realizó un análisis de componentes principales (ACP), se considera el crecimiento alométrico, todas las medidas fueron transformadas a logaritmo, el método de Burnaby (Burnaby 1966) se usó para la corrección de la talla (McCoy et al. 2006) de los 23 caracteres morfométricos (Cuadros 1, 2); para analizar la formación de grupos dentro de las poblaciones de Hemibrycon, se comparó cada población con las especies registradas de Hemibrycon (H. boquiae y H. rafaelense). En el ACP sólo fueron procesados los ejemplares que tuvieron una longitud estándar mayor o igual a $45 \mathrm{~mm}$, que es la talla de madurez sexual mínima para $H$. boquiae (Román-Valencia et al. 2008).

Se tomó la media aritmética como medida de tendencia central para la morfometría y los valores máximos y mínimos correspondientes a cada carácter. Para la merística se aplicó un análisis de varianza (ANDEVA) con un nivel de significancia de 0.05 y una prueba Tukey, para corroborar la presencia de diferencias interespecificas estadísticamente significativas.

Se diseñó una matriz de caracteres osteológicos, los caracteres se codificaron como binarios y se presentan en: descripción de caracteres; se realizó un análisis cluster exploratorio con el algoritmo paired group y la medida de similaridad de Jaccard. Se usaron 29 de 81 caracteres osteológicos y un carácter merístico, que presenta variación entre las poblaciones. Para evaluar los agrupamientos presentados por el clusters se realizaron gráficos de Andrews. Para el procesamiento de datos se utilizó el software Past, versión 1.28 de 2004 y Sigma Plot versión 10.0 de 2006.

\section{Material examinado}

Hemibrycon boquiae: Colombia, Quindío; IUQ 543, 18; Salento, vereda La Nubia, alto Cauca, cuenca del río Quindío, quebrada Las Águilas sobre la vía San Juan-a Nubia, 100 m 


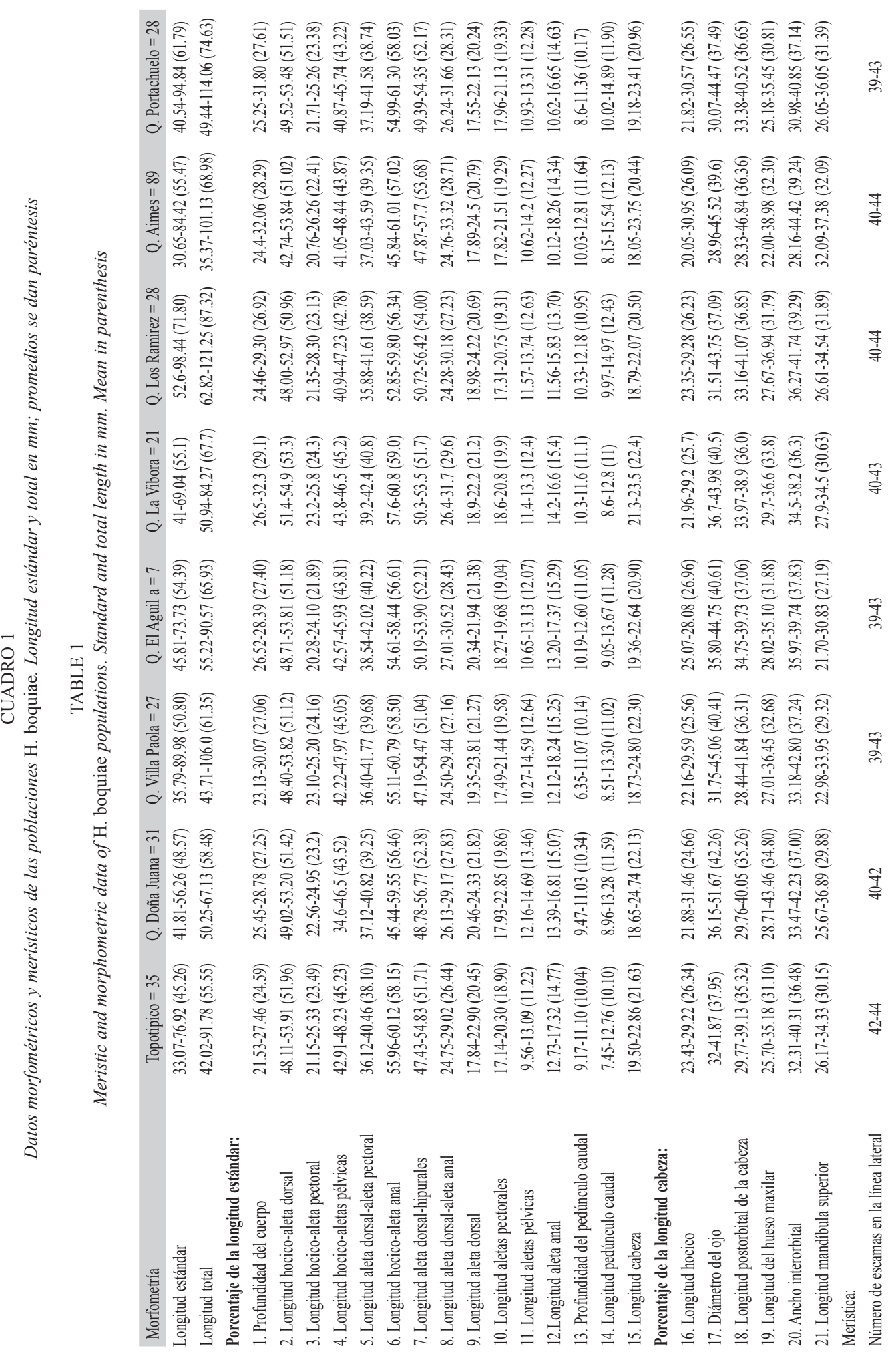




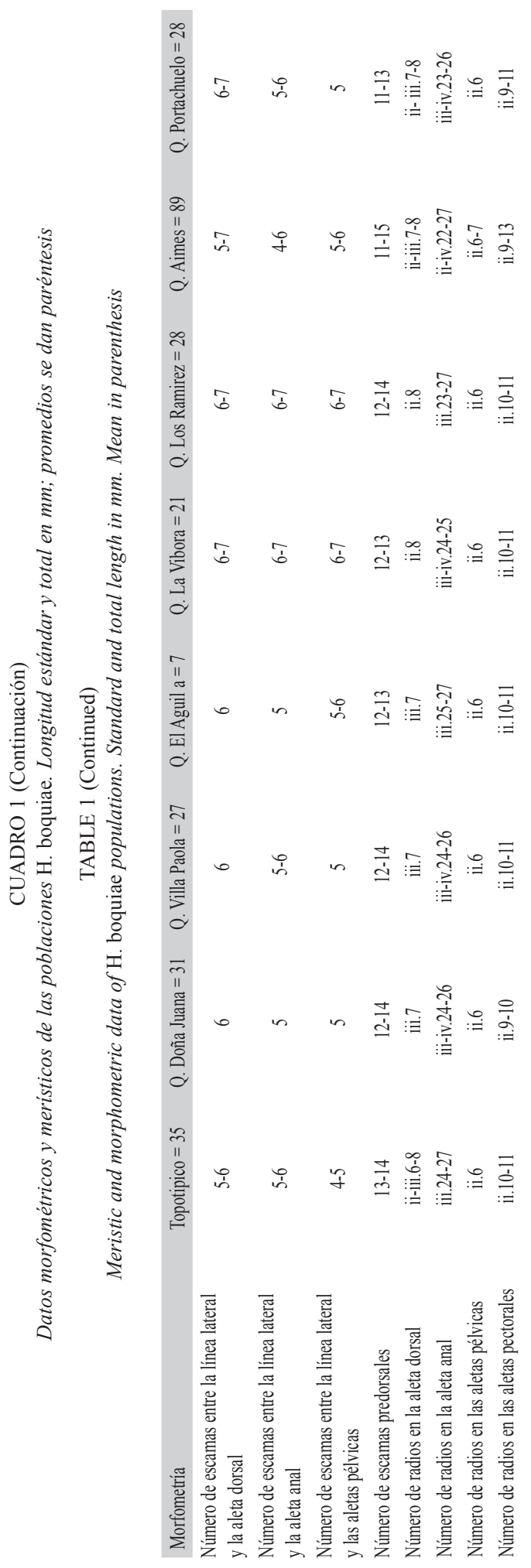

antes del puente sobre el río Quindío (4³6'04" $\mathrm{N}$ y $75^{\circ} 37^{\prime} 17^{\prime \prime}$ W) a $1601 \mathrm{msnm}, 30$ mayo 2004. Colombia, Quindío; IUQ 545, 5; Salento, vereda La Nubia, alto Cauca, cuenca del río Quindío, quebrada Las Águilas sobre la vía San Juan-La Nubia, $100 \mathrm{~m}$ antes del puente sobre el río Quindío (4³6’04" N y 75³7'17” W) a 1 601 msnm, 30 mayo 2004. Colombia, Quindío; IUQ 546, 9; Salento, vereda La Nubia, alto Cauca, cuenca del río Quindío, quebrada Doña Juana $500 \mathrm{~m}$ antes de la quebrada La Víbora sobre la vía San Juan-Boquia (4³7'39” N y 75'35'54" W) 1714 msnm, 30 mayo 2004. Colombia, Quindío; IUQ 548 (a), 25; Salento, finca Villa Paola, sobre la vía a Boquia, alto Cauca, cuenca del río Quindío, quebrada Villa

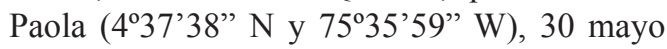
2004. Colombia, Quindío; IUQ 548 (b), 16; Salento, vereda La Nubia, alto Cauca, cuenca del río Quindío, quebrada Doña Juana $500 \mathrm{~m}$ antes de la quebrada La Víbora sobre la vía San Juan-Boquia (4³7'39” N y 75³5'54" W) 1714 msnm, 3 junio 2004. Colombia, Quindío; IUQ 549, 16; vereda El Agrado, finca, alto Cauca, cuenca del río Quindío, quebrada Doña Juana $500 \mathrm{~m}$ antes de la quebrada La Víbora sobre la vía San Juan-Boquia (4³7'39" N y 75'35'54" W) $1714 \mathrm{msnm}, 30$ mayo 2004. Colombia, Quindío; IUQ 551; Salento, vereda el agrado, finca Villa Paola, sobre la vía a Boquia, alto Cauca, cuenca del río Quindío, quebrada Villa Paola (43' $38^{\circ}$ " N y 75\%35'59" W), 30 mayo 2004. Colombia, Quindío; IUQ 871, 15; quebrada Boquia, vereda Boquia, afluente río Quindío; 26 marzo 2002. Colombia, Quindío; IUQ 301a, 3 (C \& T); quebrada Boquia, afluente río Quindío vereda Boquia, debajo del puente en la vía Boquia, 30 septiembre 1998. Colombia, Quinchia; IUQ 536, 4 (C \& $\mathrm{T})$; Vereda Opirama, río Opirama, quebrada

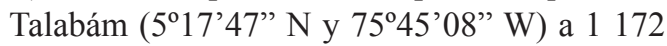
msnm, 18 octubre 2004. Colombia, Caldas; IUQ 491, 72; Neira, Tareas, medio Cauca, río Tareas $100 \mathrm{~m}$ en la vía Neira-Salamina (5'12’04” N y 75³2'56" W) 1286 msnm; 30 noviembre 2002. Colombia, Caldas; IUQ 493, 1; Neira, Tareas, medio Cauca, quebrada Aimes, río Tareas $100 \mathrm{~m}$ en la vía Neira-Salamina 
CUADRO 2

Datos morfométricos y merísticos en poblaciones de H. rafaelense. Longitud estándar y total en mm; promedios en paréntesis

TABLE 2

Meristic and morphometric data of $\mathrm{H}$. rafaelense populations. Standard and total length in mm. Mean in parenthesis

\begin{tabular}{|c|c|c|c|}
\hline Morfometria & Q. San Rafael $=50$ & Q. San Jose $=18$ & Q. Canceles $=28$ \\
\hline Longitud estándar & $22.73-89.95(40.91)$ & $37.28-74.64(59.66)$ & $40.02-84.28(56.43)$ \\
\hline Longitud total & $28.29-105.6(50.26)$ & $46.78-91.46(73.33)$ & $49.47-101.83(67.04)$ \\
\hline \multicolumn{4}{|l|}{ Porcentaje de la longitud estándar: } \\
\hline 1. Profundidad del cuerpo & $23.27-29.87(26.22)$ & $24.98-28.69(26.92)$ & $25.17-32.4(29.08)$ \\
\hline 2. Longitud hocico-aleta dorsal & $48.33-54.24(51.31)$ & $49.82-53.44(51.23)$ & $50.11-55.35(51.97)$ \\
\hline 3. Longitud hocico-aleta pectoral & $21.26-25.84(23.81)$ & $21.48-23.87(22.74)$ & $20.7-30.17(23.87)$ \\
\hline 4. Longitud hocico-aletas pélvicas & $42.00-46.74(44.52)$ & $41.41-45.36(43.30)$ & $41.56-46.26(43.64)$ \\
\hline 5. Longitud aleta dorsal-aleta pectoral & $35.21-41.26(38.23)$ & $35.78-41.08(38.71)$ & $36.45-43.14(39.67)$ \\
\hline 6. Longitud hocico-aleta anal & $54.74-59.92(56.99)$ & $54.92-58.32(56.85)$ & $54.72-62.25(58.29)$ \\
\hline 7. Longitud aleta dorsal-hipurales & $46.33-55.49(52.20)$ & $48.93-58.64(53.62)$ & $48.14-54.62(51.77)$ \\
\hline 8. Longitud aleta dorsal-aleta anal & $23.78-30.75(28.00)$ & $26.31-30.71(27.83)$ & $26.96-32.42(29.95)$ \\
\hline 9. Longitud aleta dorsal & $17.39-23.76(21.68)$ & $18.83-23.68(20.43)$ & $19.09-23.98(21.93)$ \\
\hline 10. Longitud aletas pectorales & $17.75-22.18(19.66)$ & $18.49-21.71(19.66)$ & $18.68-22.54(20.33)$ \\
\hline 11. Longitud aletas pélvicas & $11.06-16.06(12.41)$ & $10.56-15.99(12.15)$ & $10.16-16.28(13.22)$ \\
\hline 12.Longitud aleta anal & $11.00-19.23(15.10)$ & $10.72-16.73(13.73)$ & $12.65-20.10(15.64)$ \\
\hline 13. Profundidad del pedúnculo caudal & $8.28-11.88(10.48)$ & $9.39-12.97(10.97)$ & $9.68-12.82(11.18)$ \\
\hline 14. Longitud pedúnculo caudal & $7.25-13.31(9.94)$ & $9.05-13.89(10.94)$ & $8.65-12.43(10.11)$ \\
\hline 15. Longitud cabeza & $18.17-23.14(22.06)$ & $19.40-24.42(21.48)$ & $20.15-24.48(21.82)$ \\
\hline \multicolumn{4}{|l|}{ Porcentaje de la longitud cabeza: } \\
\hline 16. Longitud hocico & $20.29-29.07(24.48)$ & $20.91-30.69(25.19)$ & $22.04-30.02(26.20)$ \\
\hline 17. Diámetro del ojo & $36.65-43.58(40.18)$ & $32.99-46.34(39.83)$ & $34.65-45.07(39.87)$ \\
\hline 18. Longitud postorbital de la cabeza & $31.21-39.9(35.44)$ & $32.52-41.4(37.47)$ & $33.10-43.17(37.57)$ \\
\hline 19. Longitud del hueso maxilar & $28.00-37.76(32.35)$ & $27.84-39.76(33.8)$ & $25.23-36.18(32.56)$ \\
\hline 20. Ancho interorbital & $34.74-41.55(37.83)$ & $34.12-43.48(38.76)$ & $32.04-42.04(36.96)$ \\
\hline 21. Longitud mandíbula superior & $24.49-35.53(29.85)$ & 29.71-37.31 (33.29) & $23.28-37.87(30.56)$ \\
\hline \multicolumn{4}{|l|}{ Merística: } \\
\hline Número de escamas en la línea lateral & $40-42$ & $40-43$ & $38-40$ \\
\hline $\begin{array}{l}\text { Número de escamas entre la línea lateral } \\
\text { y la aleta dorsal }\end{array}$ & $6-7$ & $5-6$ & $5-7$ \\
\hline $\begin{array}{l}\text { Número de escamas entre la línea lateral } \\
\text { y la aleta anal }\end{array}$ & $4-6$ & $5-6$ & $5-6$ \\
\hline $\begin{array}{l}\text { Número de escamas entre la línea lateral } \\
\text { y las aletas pélvicas }\end{array}$ & $4-6$ & $5-6$ & $5-6$ \\
\hline Número de escamas predorsales & $10-12$ & $11-13$ & $10-12$ \\
\hline Número de radios en la aleta dorsal & ii-iii.7-8 & iii.7 & iii.7 \\
\hline Número de radios en la aleta anal & iii-iv.24-28 & iii-iv.24-27 & iii-v.22-27 \\
\hline Número de radios en las aletas pélvicas & ii.6 & ii.6 & ii.6 \\
\hline Número de radios en las aletas pectorales & ii.9-11 & ii. 10 & ii.9-11 \\
\hline
\end{tabular}


(5¹2’04” N y 75॰32'56” W) 1286 msnm; 28 diciembre 1995. Colombia, Caldas; IUQ 494, 3; Neira, Tareas, medio Cauca, río Tareas 100 $\mathrm{m}$ en la vía Neira- Salamina (5²'04” N y 75³2'56” W) 1286 msnm; 28 diciembre 1995. Colombia, Caldas; IUQ 530, 6, Salamina, La India, río Chamberri $\left(5^{\circ} 24^{\prime} 55^{\prime \prime} \mathrm{N}\right.$ y $75^{\circ} 30^{\prime} 10^{\prime \prime}$ W). Colombia, Honda; IUQ 531, 71; río Honda en la vía Honda a Aranzazu-Manizales, Aranzazu. Colombia, Quindío; IUQ 2387, 39; Caicedonia, sector del Alambrado, alto Cauca, cuenca del río La Vieja, quebrada Las Honda en la finca Cristales; 13 diciembre 2008.

H. rafaelense: Colombia, Risaralda; IUQ 1144, 17; río San José, 200 m vía La FloridaPereira, Risaralda, cuenca del río Otún, 12 enero de 2007. Colombia, Risaralda; ICNMHN 6703, 1; Apia, quebrada San Rafael, Sistema río Apia, en la vía Santuario-Apia, 12 abril 1991. Colombia, Risaralda; ICNMHN 3505, 50; Apia, quebrada San Rafael, Sistema río Apia, en la vía Santuario-Apia, 12 abril 1991. Colombia, Risaralda; IUQ 509, 27; Apia, quebrada San Rafael en bocas del río Apia, a $100 \mathrm{~m}$ de la vía Santuario-Apia (504'54" N y $75^{\circ} 56^{\prime} 36^{\prime}$ "W) 1 $253 \mathrm{msnm}, 8$ julio 2003. Colombia, Risaralda; MCNG 54101, 5; Apia, quebrada San Rafael en bocas del río Apia, a $100 \mathrm{~m}$ de la vía SantuarioApia (5॰04'54" N y 7556’36” W) 1253 msnm, 8 julio 2003. Colombia, Quindío; IUQ 1212, 2 (C \& T); quebrada Canceles, afluente del río La Vieja, Reserva El Ocaso, Quindío (40³3'4" $\mathrm{N}$ y 7552'71" W), 999 msnm, 3 noviembre 2006. Colombia, Quindío; IUQ 1213, 2 (C \& T); quebrada Canceles, afluente del río $\mathrm{La}$ Vieja, Reserva El Ocaso, Quindío (40³3'4" N y 7552'71” W), 999 msnm, 31 julio 2006. Colombia, Quindío; IUQ 1147, 11; quebrada Canceles, afluente del río La Vieja, Reserva El Ocaso (40³3'4" N y 7552'71' W) 999 msnm, 3 noviembre 2006. Colombia, Quindío; IUQ 1145, 20; quebrada Canceles, afluente del río La Vieja, Reserva El Ocaso, Quindío (40³3'4" N y 7552'71” W) 999 msnm, 31 julio 2006. Colombia, Quindío; IUQ 911, 3; Colombia, Quindío, Montenegro, camino a Samaria, vereda El Gigante, quebrada Canceles, afluente del río La Vieja (40³3'4” N y 7552’71” W) 999 msnm; 3 mayo 2006.

\section{RESULTADOS}

Caracteres morfométricos: La cabeza y los ojos de $H$. boquiae es pequeña (18.65$24.80 \%$ y $28.96-51.67 \%$ de longitud estándar respectivamente) y postorbital alargado (28.33$46.84 \%$ de longitud de la cabeza) (Cuadro 1). El análisis de componentes principales para los caracteres morfométricos determina poblaciones de $H$. boquiae en las quebradas: Las Águilas, Doña Juana, Villa Paola, Portachuelo, La Víbora, Aimes y Ramírez, las cuales aparecen agrupadas dentro de la variabilidad de la población topotípica de $H$. boquiae (=quebrada Boquia, Salento, Quindío) (Cuadros 3, 4; Fig. 1).

La cabeza y los ojos de $H$. rafaelense es pequeña $(18.17-24.42 \%$ y $32.99-46.34 \%$ de longitud estándar respectivamente), y postorbital alargado (31.21-43.17\% de longitud de la cabeza) (Cuadro 2). El análisis de componentes principales para los caracteres morfométricos determina poblaciones de $H$. rafaelense en las quebradas Canceles, La Clara, San Rafael y San José, agrupadas dentro de la variabilidad de la población topotípica de $H$. rafaelense (=quebrada San Rafael, alto Cauca) (Fig. 2).

Al examinar los valores propios del análisis de componentes principales ACP (Cuadro 3) se indica que los primeros cinco valores propios son significativos para $H$. boquiae y $H$. rafaelense. La estimación de la variabilidad acumulada para $H$. boquiae es $28.34 \%, 49.09 \%$, $59.99 \%, 69.35 \%$ y $75.91 \%$; para $H$. rafaelense es $25.04 \%, 44.13 \%, 58.27 \%, 66.77 \%$ y $74.17 \%$ respectivamente (Cuadro 3).

Caracteres merísticos: El ANDEVA presentó diferencias significativas en el número de escamas entre la línea lateral-aleta anal para las poblaciones de $H$. boquiae de las quebradas La Víbora y Los Ramírez (6 a 7 vs. 4 a $6, \mathrm{~F}=30.82 ; \mathrm{p}<0.000)$ y en el número de escamas entre la línea lateral-aleta pélvica para las poblaciones de las quebradas Las Águilas, 
CUADRO 3

Valor propio, obtenido a partir del análisis de componentes principales para las poblaciones de Hemibrycon boquiae $y \mathrm{H}$. rafaelense

TABLE 3

Value itself, obtained from the Principal Component Analysis (PCA) of Hemibrycon boquiae and H. rafaelense populations

\begin{tabular}{|c|c|c|c|c|}
\hline \multirow{2}{*}{$\mathrm{CP}$} & \multicolumn{2}{|c|}{ Hemibrycon boquiae } & \multicolumn{2}{|c|}{ Hemibrycon rafaelense } \\
\hline & valor propio & $\%$ varianza & valor propio & $\%$ varianza \\
\hline 1 & 0.0133109 & 28.344 & 0.0123399 & 25.04 \\
\hline 2 & 0.00974866 & 20.758 & 0.00941076 & 19.097 \\
\hline 3 & 0.00512237 & 10.907 & 0.00696818 & 14.14 \\
\hline 4 & 0.00439674 & 93.622 & 0.00418884 & 85.001 \\
\hline 5 & 0.00308479 & 65.686 & 0.00368643 & 74.806 \\
\hline 6 & 0.0022992 & 48.958 & 0.00288511 & 58.545 \\
\hline 7 & 0.00180569 & 38.449 & 0.00206002 & 41.803 \\
\hline 8 & 0.00126263 & 26.886 & 0.00178781 & 36.279 \\
\hline 9 & 0.00123428 & 26.282 & 0.00149279 & 30.292 \\
\hline 10 & 0.00104734 & 22.302 & 0.00133965 & 27.184 \\
\hline 11 & 0.000897894 & 19.119 & 0.000749306 & 15.205 \\
\hline 12 & 0.000736173 & 15.676 & 0.000552743 & 11.216 \\
\hline 13 & 0.000602836 & 12.836 & 0.000507963 & 10.308 \\
\hline 14 & 0.000337975 & 0.71967 & 0.000354649 & 0.71966 \\
\hline 15 & 0.000281348 & 0.59909 & 0.000278435 & 0.56501 \\
\hline 16 & 0.000258452 & 0.55033 & 0.000219461 & 0.44533 \\
\hline 17 & 0.000161755 & 0.34443 & 0.000155075 & 0.31468 \\
\hline 18 & 0.000133794 & 0.28489 & 0.000143514 & 0.29122 \\
\hline 19 & 0.0000973062 & 0.2072 & 0.0000803779 & 0.1631 \\
\hline 20 & 0.0000892605 & 0.19007 & 0.0000386474 & 0.078424 \\
\hline 21 & 0.0000376691 & 0.080211 & 0.0000232888 & 0.047258 \\
\hline
\end{tabular}

$\mathrm{CP}$ : componente principal. CP: principal component.

Doña Juana, Villa Paola, Portachuelo y Aimes ( 6 a 7 vs. 5 a $6, F=401.3 ; p<0.000$ ). Los demás caracteres morfométricos y merísticos de las poblaciones de $H$. boquiae de las quebradas La Víbora y Los Ramírez, se solapan con las demás poblaciones registradas (Cuadro 1).

Para $H$. rafaelense de la quebrada San Rafael, se registraron diferencias en el número de escamas entre la línea lateral- aleta pélvica (4 a 6 vs. 5 a $6, F=1.50 ; \mathrm{p}<0.000$ ) y en el número de escamas entre la línea lateral- aleta anal ( 4 a 6 vs. 5 a $6, \mathrm{~F}=2.79 ; \mathrm{p}<0.000$ ), sin embargo de acuerdo el ANDEVA estas diferencias no son estadísticamente significativas. Los demás caracteres morfométricos y merísticos de la población de $H$. rafaelense de la quebrada San Rafael, se enmascaran con las demás poblaciones.

Las poblaciones de $H$. boquiae presentan entre 40 y 44 escamas con poros en la línea lateral, inusualmente 39 escamas se registran en las poblaciones de la quebrada 
CUADRO 4

Vectores propios, obtenidos a partir del análisis de componentes principales de las poblaciones de Hemibrycon boquiae $y \mathrm{H}$. rafaelense

TABLE 4

Vectors itself, obtained from the Principal Component Analysis (PCA) of Hemibrycon boquiae and $\mathrm{H}$. rafaelense populations

\begin{tabular}{|c|c|c|c|c|}
\hline \multicolumn{5}{|l|}{ Vectores propios } \\
\hline \multirow{2}{*}{ Medidas } & \multicolumn{2}{|c|}{ Hemibrycon boquiae } & \multicolumn{2}{|c|}{ Hemibrycon rafaelense } \\
\hline & CP 2 & CP 3 & CP 2 & CP 3 \\
\hline Longitud estándar & 0.03383 & -0.01978 & 0.01944 & -0.02674 \\
\hline Longitud total & 0.04215 & -0.02326 & 0.01396 & -0.03037 \\
\hline 1. Profundidad del cuerpo & 0.1688 & -0.03383 & 0.01946 & -0.1217 \\
\hline 2. Longitud hocico-aleta dorsal & 0.02585 & -0.02495 & 0.03548 & -0.07727 \\
\hline 3. Longitud hocico-aleta pectoral & -0.06694 & 0.04361 & 0.01413 & -0.05743 \\
\hline 4. Longitud hocico-aletas pélvicas & -0.0009862 & -0.029 & 0.05212 & -0.0635 \\
\hline 5. Longitud aleta dorsal-aleta pectoral & 0.1567 & -0.06819 & 0.02158 & -0.1059 \\
\hline 6. Longitud hocico-aleta anal & 0.09599 & -0.06862 & 0.0252 & -0.05613 \\
\hline 7. Longitud aleta dorsal-hipurales & 0.1222 & -0.04572 & 0.04153 & -0.04351 \\
\hline 8. Longitud aleta dorsal-aleta anal & 0.01589 & 0.04482 & -0.0433 & -0.191 \\
\hline 9. Longitud aleta dorsal & 0.07316 & -0.05318 & -0.06393 & -0.1691 \\
\hline 10. Longitud aletas pectorales & 0.08821 & -0.02803 & 0.01657 & -0.1427 \\
\hline 11. Longitud aletas pélvicas & 0.03698 & 0.1497 & -0.06503 & -0.1821 \\
\hline 12.Longitud aleta anal & -0.02042 & -0.02011 & -0.1697 & -0.3853 \\
\hline 13. Profundidad del pedúnculo caudal & 0.229 & -0.03302 & -0.1995 & -0.2098 \\
\hline 14. Longitud pedúnculo caudal & 0.7052 & 0.1368 & -0.4171 & 0.6121 \\
\hline 15. Longitud cabeza & -0.066 & 0.007755 & -0.02213 & -0.03951 \\
\hline 16. Longitud hocico & -0.3256 & -0.6917 & -0.2645 & 0.3214 \\
\hline 17. Diámetro del ojo & -0.209 & 0.2476 & 0.09376 & 0.004032 \\
\hline 18. Longitud postorbital de la cabeza & -0.09614 & -0.09212 & 0.02569 & -0.2872 \\
\hline 19. Longitud del hueso maxilar & -0.3279 & 0.6016 & 0.4978 & 0.1308 \\
\hline 20. Ancho interorbital & 0.05562 & 0.03742 & 0.03633 & -0.01088 \\
\hline 21. Longitud mandíbula superior & -0.2753 & 0.1529 & 0.6403 & 0.2523 \\
\hline
\end{tabular}

CP: componente principal. PC: Principal Component.

Villa Paola y Águila, afluentes río Quindío y Portachuelo afluente río Roble en alto Cauca. Las poblaciones de $H$. rafaelense presentan entre 40 y 43 escamas con poros en la línea lateral, poco frecuente 38 escamas se registra en la población de la quebrada Canceles. En $H$. rafaelense la línea lateral es completa, en $H$. boquiae se observa variación: completa, incompleta ( $\mathrm{n}=3$ quebrada Boquia), interrumpida ( $\mathrm{n}=2$ quebrada Boquia) y duplicada sobre pocas escamas ( $\mathrm{n}=3$ quebrada Boquiae).

Las poblaciones de $H$. boquiae y $H$. rafaelense presentaron entre cuatro y seis dientes en la fila externa del premaxilar, con una a tres cúspides; cuatro dientes en la fila interna del premaxilar, a veces cinco dientes en ejemplares de $H$. boquiae de la quebrada Boquia (4 de 34 ejemplares) con cinco cúspides. Tres dientes grandes sobre cada dentario, seguidos de un diente mediano y de cinco a nueve dientes pequeños. Cuatro a 13 dientes extendidos en el borde ventral del maxilar en $H$. boquiae y ocho a 13 en $H$. rafaelense y multicúspides.

En $H$. boquiae se observa ausencia de simetría bilateral en los dientes del maxilar en ejemplares de la quebrada Boquia (excepto 

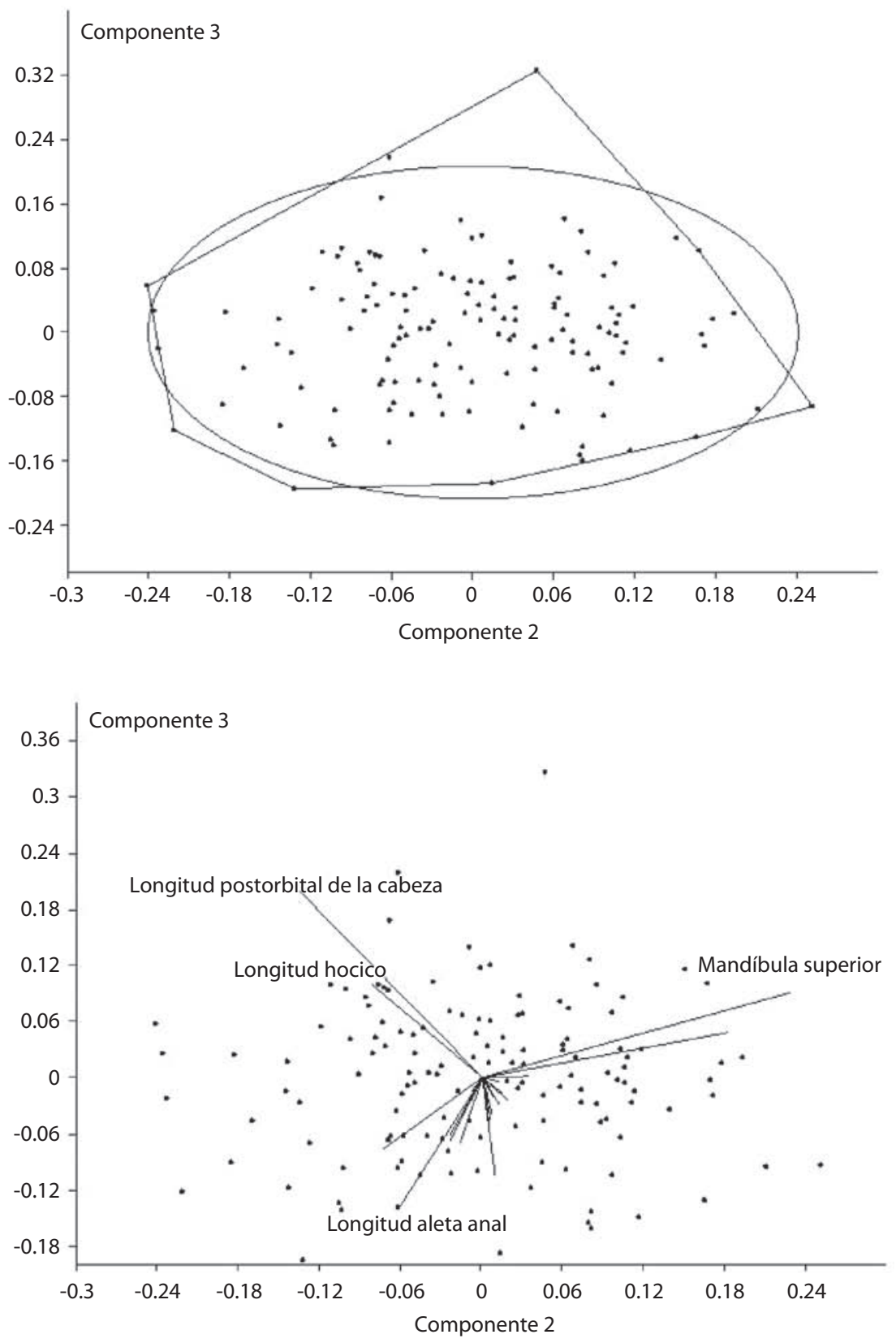

Fig. 1. Análisis de Componentes Principales (ACP) para los caracteres morfométricos de las poblaciones de H. boquiae.

Fig. 1. Principal Components Analysis (PCA) by morphometric characters of H. boquiae populations. 

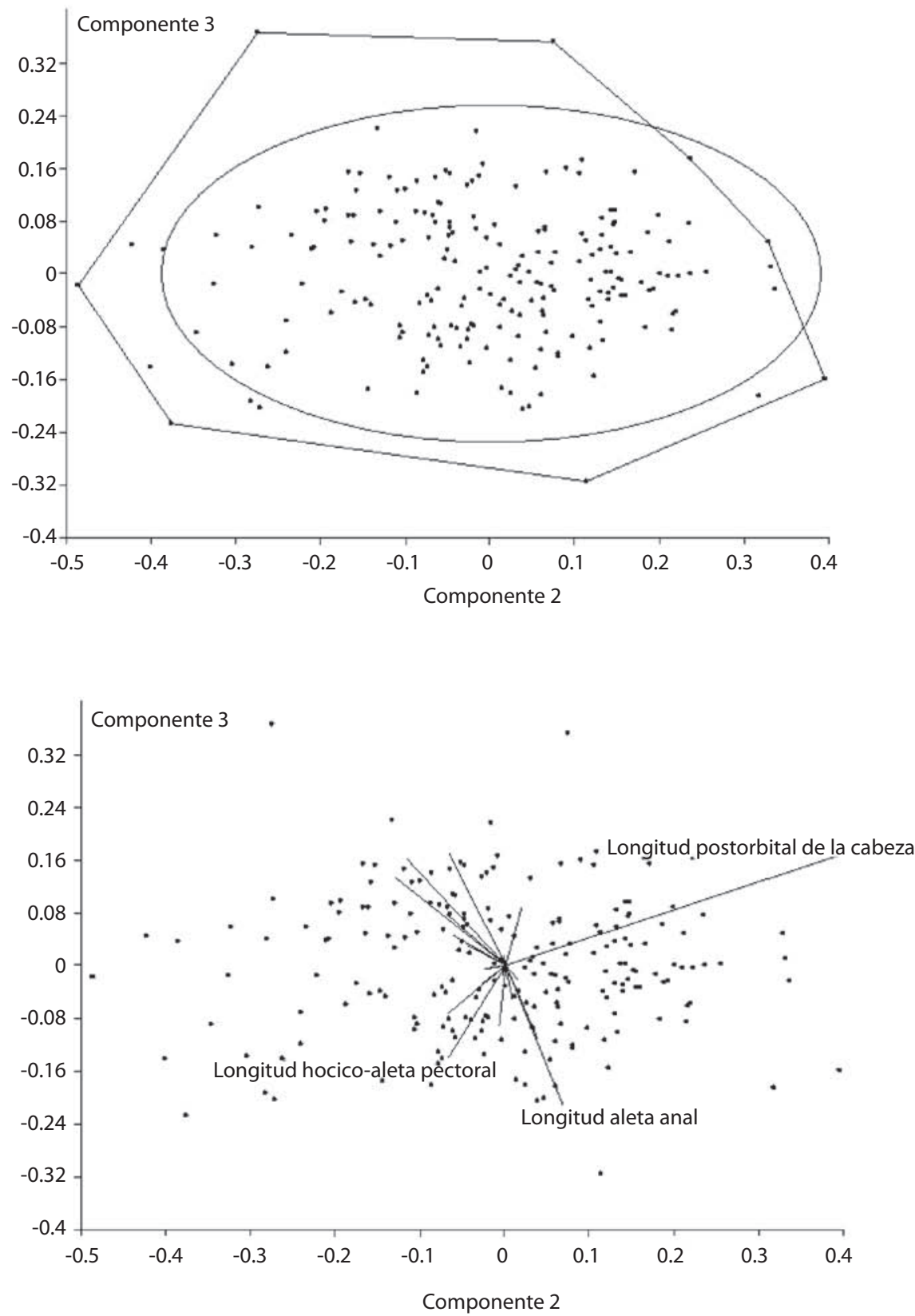

Fig. 2. Análisis de Componentes Principales (ACP) para los caracteres morfométricos de las poblaciones de H. rafaelense.

Fig. 2. Principal Components Analysis (PCA) by morphometric characters of H. rafaelense populations. 
cinco de 30 ejemplares). Sin embargo, la prueba Kruskal-Wallis mostró que no existen diferencias significativas entre el número de dientes del lado izquierdo y derecho del maxi$\operatorname{lar}(\mathrm{H}=0.007 ; \mathrm{Hc}=0.008 ; \mathrm{p}=0.92)$ (Bonferroni corregido $=0.9352)$.

Caracteres osteológicos: La distancia de similaridad del análisis cluster para los caracteres osteológicos, soporta dos grupos que corresponden con las poblaciones de $H$. boquiae y $H$. rafaelense ( $48 \%$ de similaridad, Fig. 3). Las poblaciones que se distribuyen en los drenajes del alto Cauca se encontraron más afines entre si que con las poblaciones del medio Cauca. Las poblaciones de $H$. boquiae de las quebradas Las Águilas y Villa Paola se hallaron más asociadas ( $78 \%$ de similaridad), a la vez con la población de la quebrada Doña
Juana (60\% de similaridad). Las poblaciones de la quebrada Boquia y Portachuelo se hallaron estrechamente asociadas $(72 \%$ de similaridad) y menos afines con las poblaciones de las quebradas Aimes, Chamberri y Talabam (48\% de similaridad). Las poblaciones de $H$. rafaelense de la quebrada San José y Clara se encontraron con una mayor similitud $(65 \%$ de similaridad), a la vez con la población de la quebrada Canceles (58\% de similaridad). La población de la quebrada San Rafael se halló menos asociada con las demás poblaciones de H. rafaelense ( $48 \%$ de similaridad).

\section{Descripción de caracteres (Cuadro 5,} Fig. 3):

1. Extremo anterior del maxilar alargado, con una expansión ventral en el punto de contacto con el premaxilar: (0) ausente; (1) presente.

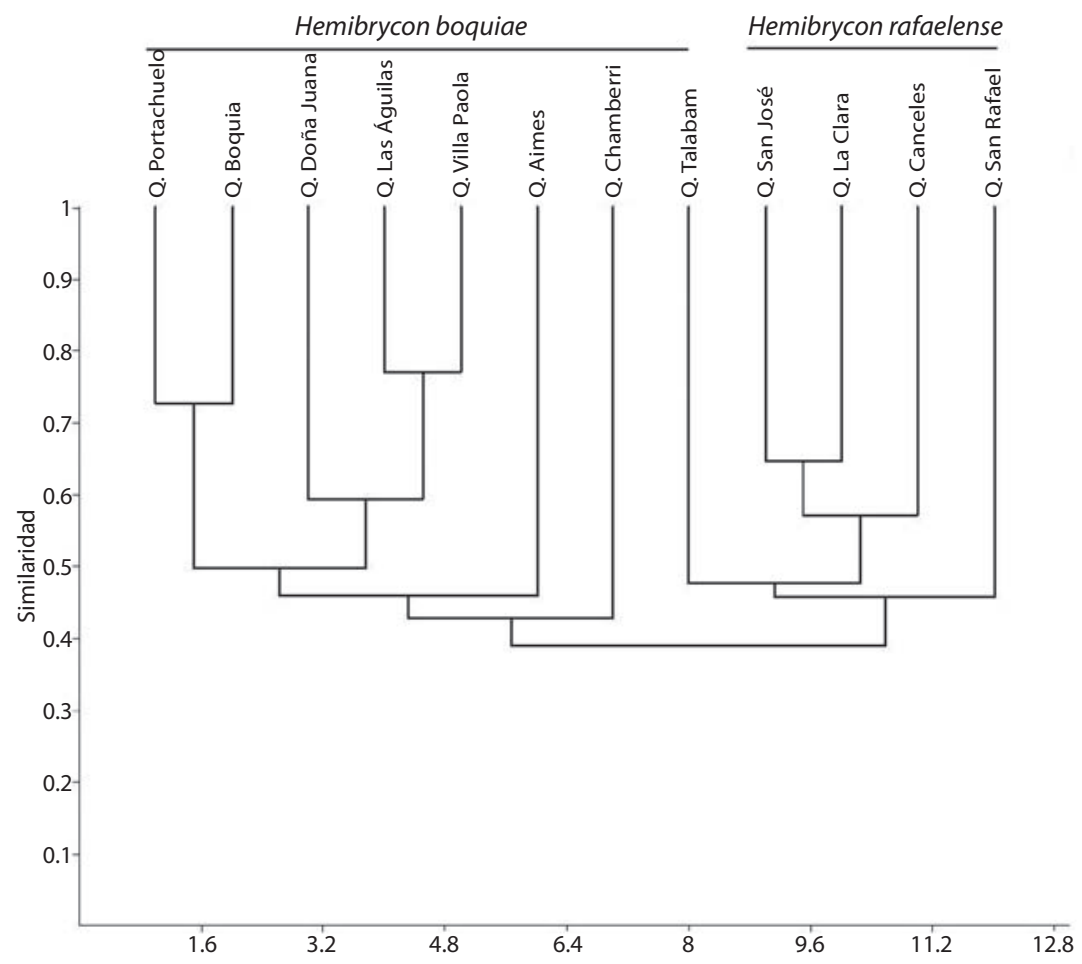

Fig. 3. Dendrograma de los caracteres osteológicos de las poblaciones de Hemibrycon boquiae y H. rafaelense, coeficiente de Jaccard.

Fig. 3. Dendrogram of osteological characters of $H$. boquiae and $H$. rafaelense populations. 


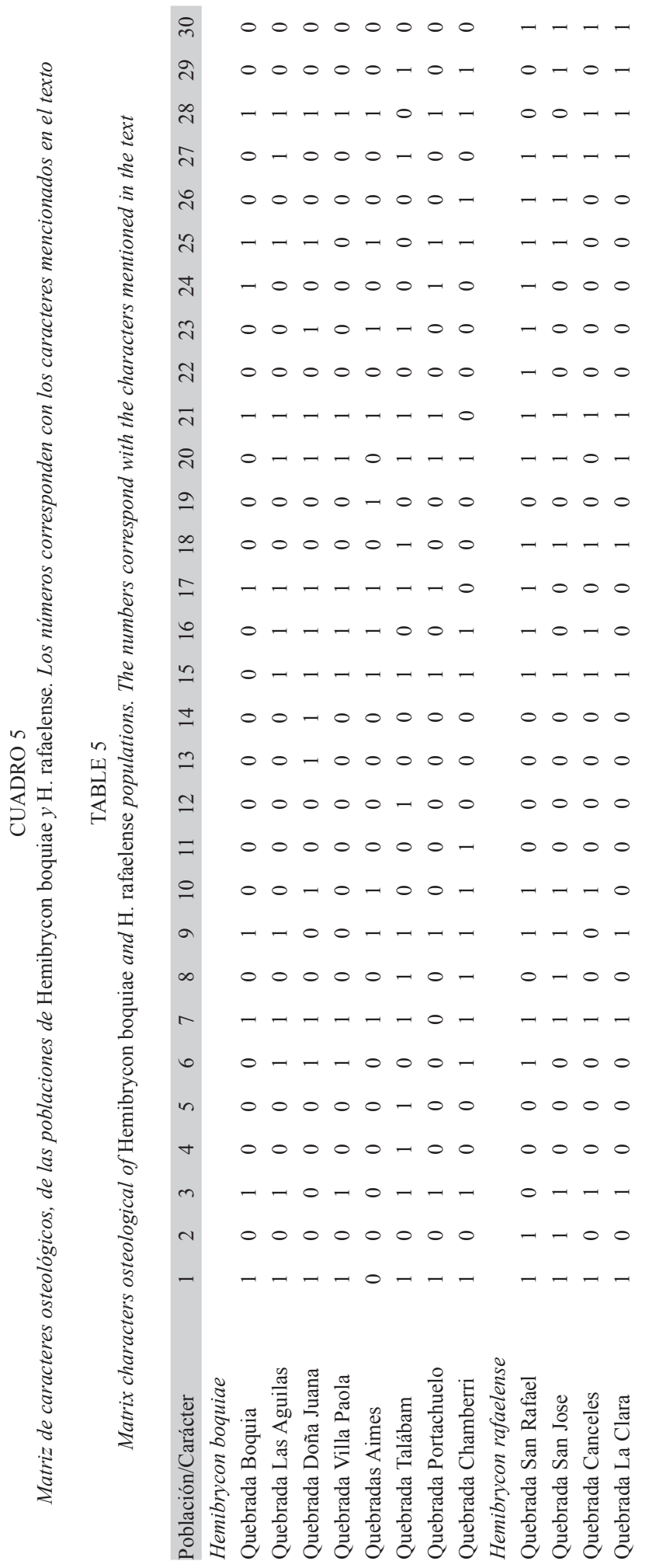


2. Número de cúspides de la fila externa del premaxilar: (0) con tres a cuatro cúspides; (1) con cinco a siete cúspides.

3. Número de dientes pequeños que siguen a los dientes grandes del dentario: $(0)$ con cinco a siete; (1) con más de siete.

4. Extremo posterior del ectoterigoide se desplaza por el margen lateral del cuadrado: (0) ausente; (1) presente.

5. Ectoterigoide: (0) en contacto con el cuadrado; (1) no en contacto con el cuadrado

6. Mesopterigoides: (0) se sobrepone sobre el metapterigoide; (1) apenas en contacto con el metapterigoide

7. Fenestra de la región posterior del metapterigoide: (0) no se completa; (1) totalmente completa.

8. Lateroetmoide alcanza a entrar en contacto con el palatino: (0) ausente; (1) presente.

9. Extremo postero ventral del antorbital, con una apófisis: (0) ausente; (1) presente.

10. Extremo postero ventral del antorbital en contacto con el extremo anterior del maxilar: (0) ausente; (1) presente.

11. Primer infraorbital en contacto con el maxilar: (0) presente; (1) ausente.

12. Canal latero sensorial del segundo infraorbital: (0) recto y con ligeras proyecciones laterales reducidas; (1) con una proyección medial de forma recta se proyecta hasta aproximadamente la mitad del infraorbital.

13. Huesos nasales presentan proyecciones laterales no uniformes: (0) ausente; (1) presente.

14. Terminación de la espina del supraoccipital: (0) de forma aguda; (1) de forma redondeada.

15. Extremo posterior del pterótico se proyecta: (0) sobre todo el extraescapular; (1) hasta la parte media del extraescapular.

16. Contacto de la espina del tercer arco neural con el complejo neural: (0) presente; (1) ausente.

17. Concavidad del coracoides, se forma al unirse con el cleitrum: (0) se extiende hasta aproximadamente la mitad del coracoides;
(1) se extiende menos de la mitad del coracoides.

18. Postcleitrum 1 y 2: (0) distantes; (1) muy cercanos

19. Postcleitrum 3: (0) presenta una proyección lateral pequeña; (1) sin proyecciones laterales.

20. Postcleitrum 1 forma una estructura: (0) corto y engrosado; (1) alargado y delgado.

21. Proceso isquial con dos proyecciones delgadas perpendiculares a su eje: (0) reducidas; (1) bien desarrolladas.

22. Radios caudales procurrentes dispuestos en el extremo ventral de la aleta caudal: (0) con seis a 11 ; (1) con más de 11 .

23. Radios caudales procurrentes dispuestos en el extremo dorsal de la aleta caudal: (0) con ocho a 10; (1) con más de 10.

24. Número de epurales: (0) constante, con dos; (1) variable, con uno a 2.

25. Número de epineurales: (0) con 36 o más; (1) con 35 o menos.

26. Número de epipleurales: (0) con 18 a 23; (1) con más de 23.

27. Número de vértebras que se extienden a partir del cuarto neural hasta el inicio de los pterigióforos de la aleta dorsal: (0) con siete a nueve; (1) con 10.

28. Primer supraneural: (0) entre el cuarto arco neural y la primera espina neural; (1) entre la primera y segunda espina neural.

29. Espinas en los radios de la aleta anal se inician a partir: $(0)$ del tercer a cuarto radio hasta el último radio de la aleta anal; (1) del quinto a sexto radio hasta el último radio de la aleta anal.

30. Número de escamas predorsales: $(0)$ con 12 a $14 ;$ (1) con 10 a 12.

\section{DISCUSIÓN}

La magnitud de las diferencias fenotípicas entre las poblaciones de Hemibrycon boquiae y $H$. rafaelense no evidenció la formación de nuevas especies, debido a que se encontraron dentro de la variabilidad y lo reportado para ambas. Las discrepancias morfológicas se hallaron principalmente asociadas a caracteres 
merísticos: como número de escamas entre la línea lateral-aleta pélvica y número de escamas entre la línea lateral-aleta anal, y osteológicos como: el número de epineurales y distancia del postcleitrum 1 con relación al postcleitrum 2 .

La variación en merística y osteología a nivel poblacional, constituye una evidencia de la plasticidad fenotípica producto de presiones selectivas, a la cual se han encontrado expuestas estas poblaciones, como la tectónica del cuaternario en el río Cauca. De acuerdo a Ives et al. (2007) las divergencias entre poblaciones de una misma especie se debe a eventos de discrepancia filogenética o efectos directos de factores ambientales entre poblaciones (plasticidad fenotípica). Otras especies, en las cuales se plantea que la variación de los caracteres merísticos en poblaciones se debe a plasticidad fenotípica son: Bryconamericus caucanus (Román-Valencia et al. en prensa) y Roeboides dayi (Lucena 2000). La plasticidad fenotípica es inducida por heterogeneidad o estrés ambiental, cambios en la temperatura, humedad o concentración de oxígeno (Garland \& Kelly 2006).

Eigenmann (1927) plantea que $H$. boquiae estáestrechamenteasociadacon Bryconamericus tolimae y con B. caucanus, además, anota la dificultad para distinguir a $H$. boquiae de $B$. caucanus. Román-Valencia (2001) afirma que el número de dientes en el maxilar, distingue a $H$. boquiae de $B$. caucanus. Sin embargo, en éste estudio, el número de dientes a lo largo del maxilar es un carácter que varia en $H$. boquiae, coincide con Eigenmann (1927) y Géry (1962). Para distinguir a $H$. boquiae de $B$. caucanus se consideran caracteres asociados a machos, es decir, las espinas reducidas en los radios de todas las aletas, menos la caudal en H. boquiae, y espinas bien desarrolladas en los radios de las aletas anal y pélvicas en $B$. caucanus. Miles (1971) registra en H. boquiae 42 a 45 escamas, con poros en la línea lateral, aquí su ámbito de variación se observa de 40 a 44 escamas (en 265 de 277 ejemplares), inusualmente 39 (en 10 de 277 ejemplares) y 45 (en 2 de 277 ejemplares) (Cuadro 1).

La distribución de $H$. boquiae, a partir de este estudio, se amplia y comprende en nueve drenajes del alto y medio Cauca, todas en alopatría. Las poblaciones de H. rafaelense se localiza en cuatro drenajes del alto Cauca. Palencia (1995) cita H. boquiae para la cuenca alta de los ríos Uribante y Doradas en Venezuela por dos caracteres: número de escamas con poros en la línea lateral (38 a 40) y número de radios ramificados en la aleta anal (27), coincide con $H$. jabonero escamas con poros de la línea lateral 38 a 42 y radios ramificados de la aleta anal 23 a 28 y su distribución. Por lo tanto, ésta identificación no corresponde con $H$. boquiae. Maldonado-Ocampo et al. (2005), Mojica et al. (2006) y Villa-Navarro et al. (2006) registran a $H$. boquiae para el alto Magdalena, lo cual es un error de determinación y de distribución. Corresponde con una nueva especie de Hemibrycon, que se diferencia de $H$. boquiae por el número de radios ramificados en la aleta anal (23 a 27 vs. 19 a 23).

\section{AGRADECIMIENTOS}

Se recibió financiación de la Universidad del Quindío-Vicerrectoría de investigaciones (proyectos 212 y 304). A Maria Dolly García (grupo de investigación y asesoría estadística de la Universidad del Quindío) por sus comentarios y discusión al procesamiento estadístico. Donald C. Taphorn corrigió el resumen en inglés.

\section{RESUMEN}

Analizamos la variación morfológica y osteológica en Hemibrycon boquiae y H. rafaelense en la cuenca del río Cauca. Los análisis multivariados determinan poblaciones de $H$. boquiae en las quebradas: Boquiae, Honda, Las Águilas, Doña Juana, Villa Paola, Portachuelo, La Víbora, Aimes y Ramírez y poblaciones de $H$. rafaelense en las quebradas: Canceles, La Clara, San Rafael y San José. No hay diferencias en la morfometría entre las poblaciones de $H$. boquiae y $H$. rafaelense. Se presentan discrepancias merísticas significativas para las poblaciones de $H$. boquiae $(\mathrm{n}=277)$ y $H$. rafaelense $(\mathrm{n}=121)$ en: número de escamas entre la línea lateral y la aleta anal, y número de escamas entre la línea lateral y la aleta pélvica. La distancia de similaridad del análisis de agrupamiento (“cluster") para los caracteres osteológicos, al igual que el número de escamas predorsales, apoyan la existencia de dos grupos discretos que corresponden a $H$. boquiae y $H$. rafaelense. 
Palabras clave: morfología, distribución, Hemibrycon, Andes, pez tropical.

\section{REFERENCIAS}

Arcila-Mesa, D.K. 2008. Análisis filogenético y biogeográfico de las especies de Hemibrycon (Characiformes, Characidae). Trabajo de Bachillerato, Universidad del Quindío, Programa de Biología, Armenia, Colombia.

Bertaco, V.A., L.R. Malabarba, M. Hidalgo \& H. Ortega. 2007. A new species of Hemibrycon (Teleostei: Characiformes: Characidae) from the río Ucayali drainage, Sierra del Divisor, Perú. Neotrop. Ichthy. 5: 251-257.

Burnaby, T.P. 1966. Growth-invariant discriminant functions and generalized distances. Biometrics 22: 96-116.

Eigenmann, C.H. 1927. The American Characidae. Mem. Mus. Comp. Zool. 43: 311-428.

Garland, Jr. T. \& S.A. Kelly. 2006. Phenotypic plasticity and experimental evolution. J. Exp. Biol. 209: 2344-2361.

Géry, J. 1962. The distribution pattern of the genus Hemibrycon, with a description of a new species from Surinam and an incursion into ecotaxonomy. Bul. Aquatic Biol. 3: 65-80.

Ives, A.R., P.E. Midford \& T. Jr. Garland. 2007. Withinspecies variation and measurement error in phylogenetic comparative methods. Syst. Biol. 56: 252-270.

Langerhans, R.B., G.A. Layman, A.K. Langerhans \& T.J. Dewitt. 2003. Habitat-associated morphological divergence in two Neotropical fish species. Biol. J. Linnean Soc. 80: 689-698.

Lima, F.C.T, L.R. Malabarba, P.A. Backup, J.F. Pezzi da Silva, R.P. Vari, A. Harold, R. Benine, O.T. Oyakawa, C.S. Pavanelli, N.A. Menezes, C.A.S. Lucena, M.C.S. Malabarba, Z.M.S. Lucena, R.E. Reis, F. Langeani, L. Cassati, V.A. Bertaco, C. Moreira \& P.H.F. Lucinda. 2003. Genera Incertae Sedis in Characidae, p.106169. In R.E. Reis, S.O. Kullander \& C.J. Ferrais Jr. (eds.). Checklist of the freshwater fishes of South and Central America. Edipucrs, Porto Alegre, Brasil.

Lucena, C.A.S. 2000. Revisão taxonômica e filogenía das espécies transandinas do gênero Roeboides Günther (Teleostei: Ostariophysi: Characiformes). Común. Mus. Ciênc. Tecnol. PUCRS, Serie Zoología Porto Alegre 13: 3-63.
Maldonado-Ocampo, J.A., A. Ortega-Lara, O. Usma, N. Galvis, F.A. Villa-Navarro, G. Vasquez, S. PradaPedreros \& R. Ardila. 2005. Peces de los Andes de Colombia. Instituto de Investigaciones de Recursos Biológicos "Alexander Von Humboldt", Bogotá, Colombia.

McCoy, M.W., B.M. Bolker, C.W. Osenberg, B.G. Miner \& J. R Vonesh. 2006. Size correction: comparing morphological traits among populations and environments. Oecologia 148: 547-554.

Miles, C. 1971. Los peces del Río Magdalena. Universidad del Tolima, Ibagué, Colombia.

Mojica, J.I., G. Galvis, P. Sánchez-Duarte, C. Castellanos \& F.A. Villa-Navarro. 2006. Peces del valle medio del río Magdalena, Colombia. Biota Colomb. 7: 23-38.

Ortega-Lara, A., J.S. Usma, P.A. Bonilla \& N.L. Santos. 2006. Peces de la cuenca alta del río Cauca, Colombia. Biota Colomb. 7: 39-54.

Palencia, P. 1995. Clave identificatoria para los peces de la cuenca alta de los ríos Uribante y Doradas, Edo. Táchira, Venezuela. Rev. Ecol. Lat. Am. 3: 1-4.

Román-Valencia, C. 2001. Redescripción de Hemibrycon boquiae (Pisces: Characidae), especie endémica de la quebrada Boquia, cuenca del Río Quindío, Alto Cauca, Colombia. Dahlia Rev. Asoc. Colomb. Ictiól. 4: 27-32.

Román-Valencia, C., C.R.I. Ruiz \& R. Barriga. 2006. Una nueva especie de pez del género Hemibrycon (Characiformes: Characidae). Rev. Biol. Trop. 54: 209-217.

Román-Valencia, C., R.I. Ruiz-C. \& R. Barriga. 2007. Redescripción de Hemibrycon orcesi Böhlke 1958 y H. polyodon Günther 1864 (Pisces: Characidae), incluye clave para las especies de Hemibrycon en Ecuador. Anim. Biodivers. Conserv. 30: 179-188.

Román-Valencia, C. \& D.K. Arcila-Mesa. 2008. Hemibrycon rafaelense (Characiformes, Characidae), a new species from the upper Cauca River, with key to Colombian species. Anim. Biodivers. Conserv. 31.1: 1-9.

Román-Valencia, C., R.I. Ruiz-C. \& A. Giraldo. 2008. Dieta y reproducción de dos especies sintópicas: Hemibrycon boquiae y Bryconamericus caucanus (Pisces: Characidae) en la quebrada Boquia, río Quindio, Alto Cauca, Colombia. Rev. Mus. Argentino Cienc. Nat., n.s. 10:55-62. 
Román-Valencia, C., J. A. Vanegas-Ríos \& M. D. García. En prensa. Variación morfológica de Bryconamericus caucanus (Teleostei: Characidae) en los ríos CaucaMagdalena y Ranchería, Colombia. Rev Mex Biodivers.

Song, J. \& L.R. Parenti. 1995. Clearing and staining whole fish specimens for simultaneous demostration of bone, cartilage and nerves. Copeia 1995: 114-118.

Taylor, W.R. \& G.C. Van Dyke. 1985. Revised procedures for staining and clearing small fishes and other vertebrates for bone and cartilage study. Cybium 9: 107-119.
Vari, R.P. 1995. The Neotropical fish family Ctenoluciidae (Teleostei: Ostaríophysi: Characiformes): supra and intrafamilial phylogenetic relationship, with a revisionary study. Smith. Contr. Zool. 564: 1-96.

Weitzman, S.H. 1962. The osteology of Brycon meeki, a generalized characid fish, with an osteological definition of the family. Stanford Ichthyol. Bull. 8: 1-77.

Villa-Navarro, F.A., P.T. Zuñiga-Upegui, D. Castro-Roa, J.E. García-Melo, L.J. García-Melo \& M.E. HerradaYara. 2006. Peces del alto Magdalena, cuenca del río Magdalena, Colombia. Biota Colomb. 7: 3-22. 